\title{
Noise optimisation of a piezoresistive CMOS MEMS for magnetic field sensing
}

\author{
Vincent Beroulle, Yves Bertrand, Laurent Latorre, Pascal Nouet \\ Laboratoire d'Informatique, de Robotique et de Microélectronique de Montpellier (France) \\ Contact author: latorre@lirmm.fr
}

\begin{abstract}
Using 100\% industrial fabrication processes, the design and the fabrication of a monolithic CMOS MEMS magnetic field sensor, targeting noise reduction, are developed in this paper. The sensor is based on a resonant cantilever structure with optimized electronics for signal treatment and noise filtering in order to achieve competitive performances. With a final sensitivity of $350 \mathrm{~V}_{\mathrm{rms}} / \mathrm{T}$ and a $2 \mu \mathrm{T}$ resolution, the system is able to measure earth natural magnetic field.
\end{abstract}

Key words: CMOS MEMS, magnetic sensor, noise

\section{INTRODUCTION}

Magnetic field sensing devices regain interest since new applications have been demonstrated for automotive systems including navigation and mechanical parts (e.g. wheels) motion detection (ABS). Because magnetic sensing does not involve mechanical contact and wear, it offers the reliability level required by security concerns.

In such applications, major concerns are reliability, performances and cost. Monolithic integration of intelligent sensing devices using a standard microelectronic process (CMOS) provides significant cost reduction leading to high volume production. Also reliability is improved by reducing the number of wired connections. Regarding performances, it is obvious that the best magnetic field sensor will be achieved using dedicated processes (e.g. GMR [1]), however, the purpose of this paper is to show that using a CMOS 
MEMS sensing device, on-chip signal treatment allows for overall performance improvement.

Electromechanical CMOS magnetic field sensors have been under investigation in our laboratory for several years [2]. The first part of the paper is dedicated to the electromechanical cell: the "U-Shape" cantilever. Sensing principle, and modeling are addressed with detail on the methodology we used to integrate the structure into a standard ASIC design flow.

The second part concerns an evaluation of the sensor performance, using a stand-alone cantilever device first, and with dedicated low-noise electronics for signal treatment. Sensitivity and resolution of overall magnetic field sensing system is finally given.

\section{THE U-SHAPE CANTILEVER DEVICE}

The "front-side bulk micromachining" (FSBM) technology is today fully industrialized and made available to fabless designers thought multi-project services for cheap prototyping [3]. This technology is based on a simple and low-cost post-process that etches selected parts of the CMOS die silicon substrate, releasing structures such as beams or membranes. Force sensing or thermal effects related applications might then be addressed using those structures.

From our point of view, monolithic MEMS is a standard mixed-signal ASIC that includes mechanical analog cells (beams, membranes...), the same way as it includes resistances or capacitors. Integrated system design is to be performed with standard EDA CAD tools including schematic capture, system level simulation and fabrication masks layout edition. Considering electromechanical blocs, this approach supposes that basic components have been previously characterized and translated into standard cells data with associated symbols, simulation models and layout synthesis helps.

Thus, in the following, the structure we used for magnetic field sensing is studied in order to provide the design environment with these required information.

\subsection{Sensing principle}

The proposed magnetic field sensor is based on the interaction between the unknown external magnetic field $B$ to be measured and a known current $I$ generated into the device itself (current loop). 


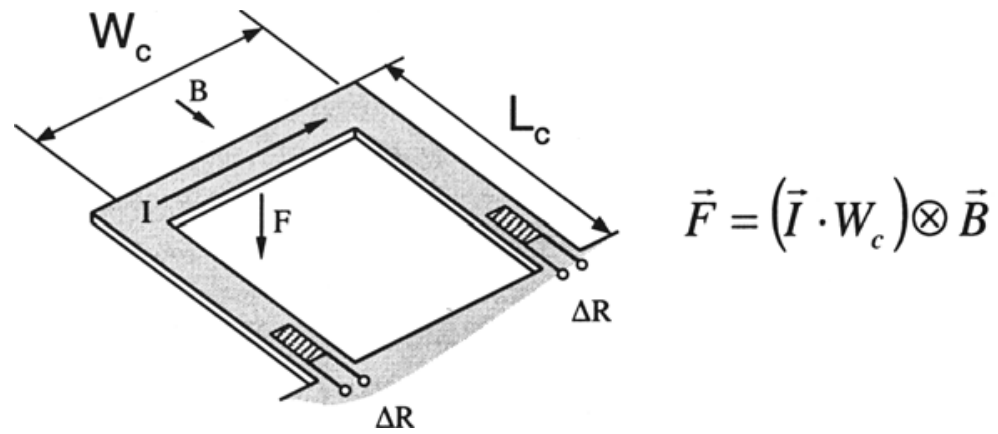

Figure 1: Magnetic field sensing principle.

The basic mechanical structure (Figure 1) consists in a U-shaped cantilever embedding an aluminum planar coil. The coil is supplied with an electrical current $I$ that can be continuous (static mode) or alternative (dynamic mode). When an external magnetic field $B$ is present, cantilever beam is therefore deflected due to the action of the Lorentz force $F$ applied to its free side. Two polysilicon strain gauges are located into the mechanical structure close to anchor points in order to detect such deflections with a maximum sensitivity.

\subsection{Modeling}

As illustrated on figure 2, the sensor is a transducer that converts a force into a resistance variation (the same variation for the two gauges).

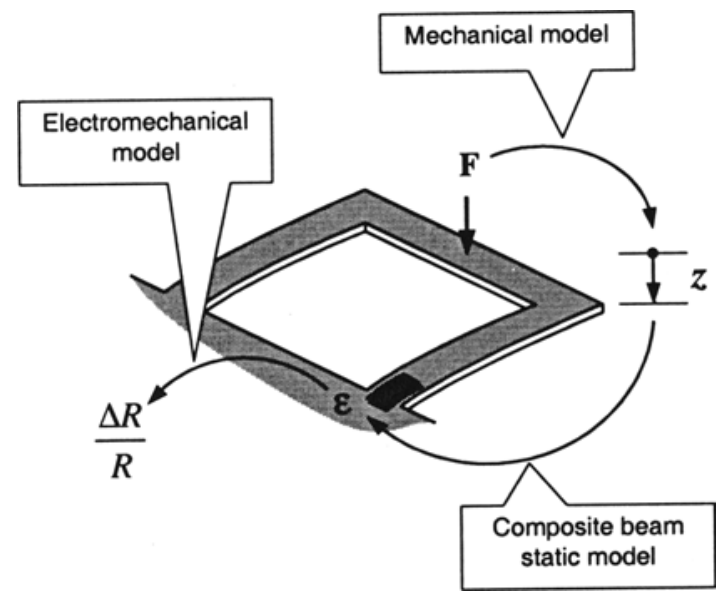

Figure 2: Modeling of the complete transducer. 
Three basic models are used in series to mathematically represent this conversion:

- A mechanical model, that describes the relation between the applied force $\mathrm{F}$ and the vertical displacement of the cantilever $\mathrm{z}$,

- A structural model for the CMOS beam that takes into account the heterogeneous composition of the structure in order to provide a relation between the vertical displacement $\mathrm{z}$ and the strain applied to the gauge,

- And finally, an electromechanical model, that translates the gauge strain into a resistance variation.

Thanks to our previous work on characterization and analytical modeling [4], we have established that the associated mechanical model is a classical second order system. Table 1 reports differential equation and Laplace transfer function where $\mathrm{k}, \mathrm{M}$ and $\mathrm{D}$ represent respectively the stiffness, the mass and the damping factor of the structure:

\begin{tabular}{|c|c|c|}
\hline \multirow{2}{*}{$M \frac{d^{2} z}{d t^{2}}=-k \cdot z-D \frac{d z}{d t}+F$} & \multicolumn{2}{|c|}{$z=S_{\text {stat }} \times \frac{B}{1+2 \cdot \xi \frac{p}{\omega_{0}}+\frac{p^{2}}{\omega_{0}{ }^{2}}}$} \\
\cline { 2 - 3 } & $\omega_{0}=\sqrt{\frac{k}{M}}$ & $\xi=\frac{D}{2 \sqrt{k M}}$ \\
\hline $\begin{array}{c}\text { Differential Equation of the mechanical } \\
\text { second order system. }\end{array}$ & $\begin{array}{c}\text { Laplace transfer function of the mechanical } \\
\text { second order system.(i) cantilever natural } \\
\text { pulsation } \omega_{0} \text {.(ii) system damping coefficient } \zeta\end{array}$ \\
\hline
\end{tabular}

Here, the vertical displacement is expressed as a function of the magnetic field $B$ and the static sensitivity $S$ stat $(\mu \mathrm{m} / \mathrm{T})$ given by:

$$
S_{\text {stat }}=\frac{I \cdot W_{c}}{k}
$$

The structure consists in an heterogeneous stack of various process layers, namely silicon oxides, metals, polysilicon and passivation nitride. In the composite beam model, the width of each layer is normalized regarding its young modulus. This operation transforms the heterogeneous beam into a equivalent homogeneous beam of equivalent Young's modulus $E_{n}$, from which we can calculate mechanical parameters such as the moment of inertia $I_{n}$ and the vertical distance $v$ between neutral axis and the gauges. 
The stiffness $k$ can be calculated from this model:

$$
k=\frac{3 \cdot E_{n} \cdot I_{n}}{L_{c}^{3}}
$$

Where $L c$ is the length of the anchored beams. Using strength of material theory, the strain in the gauge is given by:

$$
\varepsilon=\frac{F \cdot L_{c}}{E_{n} \cdot I_{n} / v}=\frac{k \cdot z \cdot L_{c}}{E_{n} \cdot I_{n} / v}=\frac{3 \cdot z \cdot v}{L_{c}^{2}}
$$

At last, piezoresistivity is a phenomenon that linearly links the strain and the material resitivity. As a result, the electromechanical model is simply:

$$
\frac{\Delta R}{R}=G f \times \varepsilon \quad \text { Where } G f \text { is called the polysilicon gauge factor. }
$$

\section{SYSTEM LEVEL INTEGRATION}

\subsection{Stand-alone cantilever performance}

While sensitivity can be useful metric, the real parameter of interest in most application is resolution. We discuss, in this part, about the minimum magnetic induction $B$ that can be measured.

Piezoresistive cantilevers suffer from an unexpected 1/f noise [5]. Unfortunately, 1/f noise in resistors from AMS process are not characterized, and corresponding spice equation is empty. So, in the following, bandwidths avoid low frequencies where $1 / \mathrm{f}$ noise can not be neglected.

White noise spectral density is constant over frequency, given for a resistor $\mathrm{R}$ about $1.7 \mathrm{k} \Omega$ by :

$$
V_{\text {noise }}^{2}(f)=4 k_{b} T R \quad V_{\text {noise,rms }}(f)=5.3 n V / \sqrt{H z}
$$

Where $\mathrm{k}_{\mathrm{b}}$ is the Boltzmann's constant, $T$ is the temperature in Kelvin and $R$ is the resistance value in Ohms. 
Two constant resistors and two gauges, equal to the same value $R$, are actually connected together in a Wheatstone bridge circuit that compensates for temperature variation. It gives a total output noise equivalent to the previous single resistor noise level.

The noise spectral density at the output of a magnetic sensor may be interpreted as a result of an noise equivalent magnetic induction (NEMI), acting on a noiseless device [6]. The following curve (figure 3 ) reports this NEMI spectral density due to White noise in resistors.

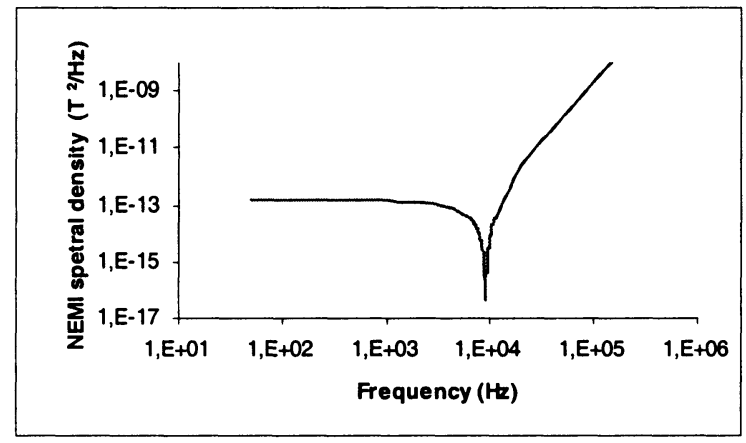

Figure 3: NEMI spectral density due to White noise in Wheatstone bridge.

Our sensor is a electro-mechanical component, thus mechanical noise must be considered. This noise is a thermal noise which can be described with a single relation giving force noise $F m$ acting on the cantilever:

$$
F m=\sqrt{4 k_{b} T D} \quad \text { With damping, } \mathrm{D}=7 \cdot 19 \cdot 10^{-7} \mathrm{~kg} \cdot \mathrm{s}^{-1}
$$

Then, this force conduces to the NEMI spectral density [6]:

$$
S_{B_{n} / U_{m e c}}(f)=\frac{4 \cdot k_{b} \cdot T \cdot D}{(W . I)^{2}}
$$

In our application, $S_{B n / U m e c}(f)$ is about $10^{-18}$, thus, mechanical noise can be neglected.

\subsection{Signal processing}

Looking at results of figure 3 , it is obvious that the best sensor performances in terms of sensitivity and resolution will be achieved using the resonant mode. The signal is then mechanically filtered and the signal 
bandwidth is restricted to the $-3 \mathrm{~dB}$ mechanical bandwidth around resonant frequency. This bandwidth is in fact only few hundreds hertz large.

It is a general rule to optimize noise performance that one should not design circuits for larger bandwidth than the signal requires. We thus designed amplification circuit and a filter in order to minimize total noise RMS value accordingly with the already restricted signal bandwidth. A schematic of the integrated circuit is presented on figure 4 .



Figure 4: Schematic of the measuring system.

In this circuit, amplification stage and bandpass filter are preceded by a multiplier, which is used to shift the amplifier input signal in the frequency domain. If the cantilever is used in resonant mode, this feature is required for adjustment purpose as long as it is difficult to precisely predict both sensor natural frequency and filter cutoff frequencies. In the case of a static actuation of the cantilever, the multiplier shall be used to shift the signal from DC to higher frequencies where the 1/f noise of amplifier becomes negligible (from the lock-in principle).

Any circuit located before amplification is under major concern concerning noise injection. For this reason, noisy operational amplifiers have been avoided in the design of the mixer. Figure 5 shows a schematic view of the Gilbert's multiplier that we used instead:

Equivalent input noise in this structure strongly depends on the differential pair transistors length. In order to find the optimal length for those transistors, a parametric noise analysis has been performed (figure 6). Because the transistors length also affects the multiplier gain ( $A$ decreases when $L$ increases) there is no benefice regarding equivalent input noise in increasing the length too much. Finally, $L$ has been fixed to $5 \mu \mathrm{m}$, resulting in a mixer gain $A$ about 10 . The layout of the integrated Gilbert's cell is presented on figure 7.

Amplification and filtering use classic, operational amplifier based, circuits. The filter is a second order band-pass filter with a cutoff frequency of $25 \mathrm{kHz}$ and a $3 \mathrm{~dB}$ bandwidth of $3 \mathrm{kHz}$. 




Figure 5: Schematic of the Gilbert's multiplier.

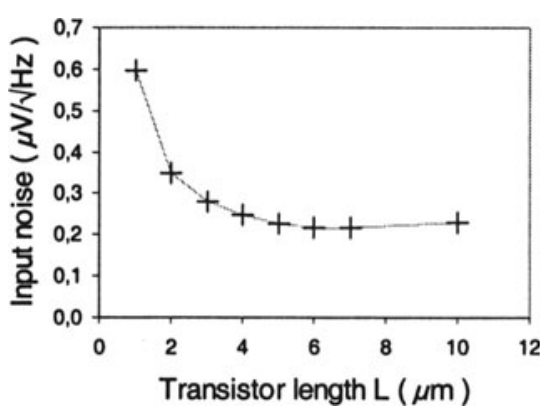

Figure 6: Equivalent input noise as a function of the differential stage transistors length.

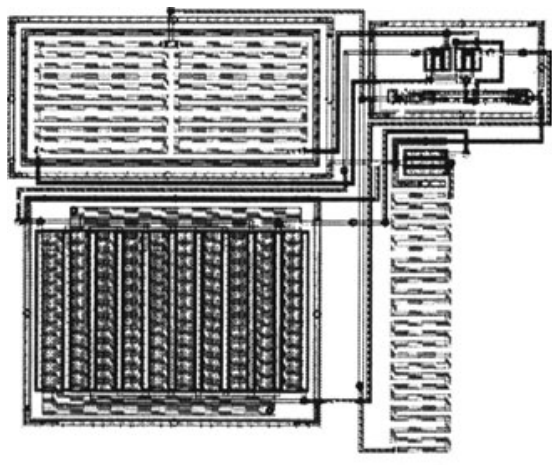

Figure 7: Layout of the multiplier

\subsection{Simulations}

In order to include the mechanical device in the design environment, the complete analytical model of the U-Shaped cantilever has been translated in Analog HDL language that authorizes simulations in a microelectronic designer framework.

This model, which is attached to the mechanical transducer, is used by analog electronic circuits simulation such as Spice. Real numbers are manipulated during the simulation regardless their physical significance. Doing this, a voltage generator can be used to generate any physical stimuli (e.g. a magnetic field) and electrical measurement (voltage or current) might represent any physical information such as displacement or speed. 
In figure 8, a magnetic field step from 0 to $1 \mathrm{mT}$ is applied to the sensor at $t=0$. From top to bottom the figure displays the following waveforms: (i) The "force input" which is the voltage source applied across the planar coil, (ii) the local oscillator control signal, (iii) the vertical displacement of the UShape in microns, (iv) the amplifier output signal and, (v) the whole system output. Spectrums have been calculated using a time window far enough form the simulation beginning, so that permanent regime as been reached.

In order to shift the $9 \mathrm{kHz}$ cantilever resonant frequency to the $25 \mathrm{kHz}$ filter central frequency, the local oscillator signal frequency has been fixed at $16 \mathrm{kHz}$. Looking at FFT's the multiplication produces the awaited frequency shift and the filter removes the undesired $6 \mathrm{kHz}$ peak.


Figure 8: Example of system level simulations.

\subsection{Noise performance analysis}

Figure 9 shows noise simulation results in terms of noise power spectral density for the complete signal processing circuit including the sensor gauges, the multiplier, the amplifier and the filter. However, as long as nonlinear circuit are not completely supported for noise analysis, the L.O. signal has been fixed to a constant level, transforming the multiplier into a simple amplification stage with a gain of 10 . Doing this, the multiplier input noise is not shifted in the frequency domain. This cperation does not affect the result 
regarding thermal noise into the sensor gauges since its noise power spectral density is constant over the frequency range.

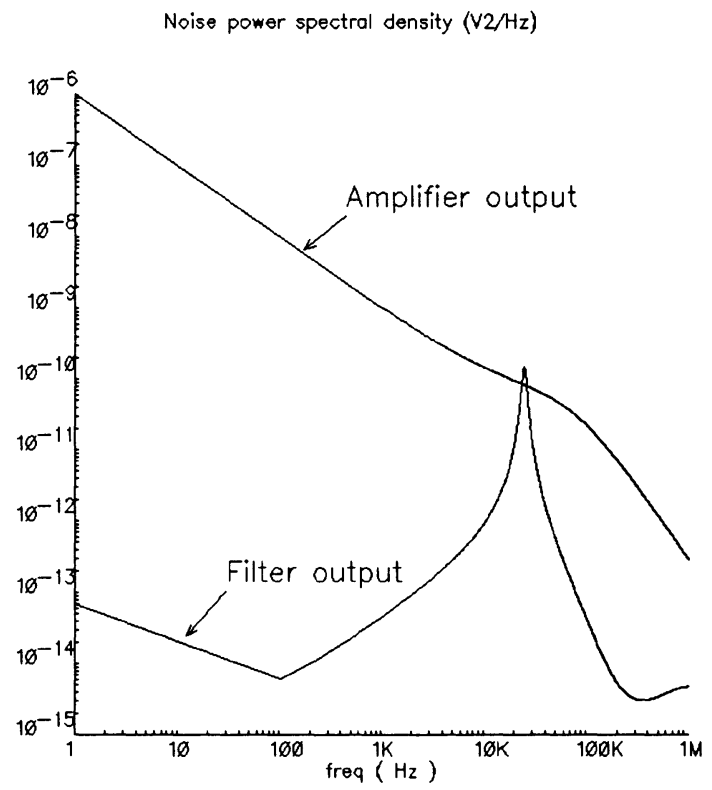

Figure 9: Noise power spectral density before and after filtering

According to simulation results, the total noise level is about $8 \mathrm{mVrms}$ before filtering and $820 \mu \mathrm{Vrms}$ when filtered with a $3 \mathrm{kHz}$ bandwidth.

Since total amplification factor with mixer and square local oscillator is 660 , the sensitivity of the resonant sensor becomes $350 \mathrm{Vrms} / \mathrm{T}$ with a $2 \mu \mathrm{T}$ resolution.

\subsection{System fabrication}

The system has been fabricated using $0.6 \mu \mathrm{m}$ CMOS technology from AMS [7]. The figure 10 presents the circuit after CMOS process. The gray square around the U-Shape device is the bare silicon defined during the mask design step by stacking all the oxides openings (diffusion, contact, via and pad). After CMOS fabrication, dies are anisotropically wet etched in a TMAH solution, releasing the cantilever as shown on figure 11 . 


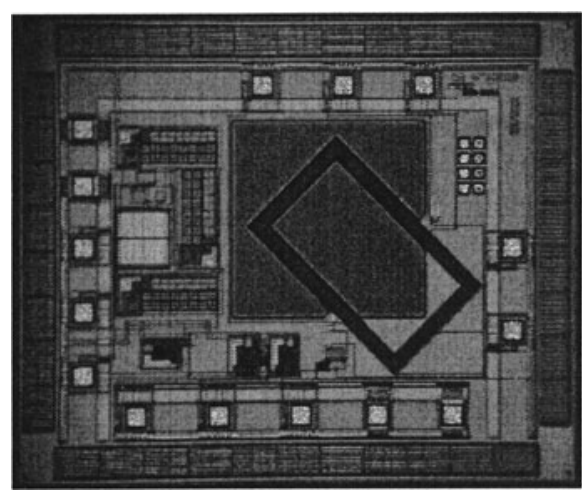

Figure 10: The sensing system after CMOS process.

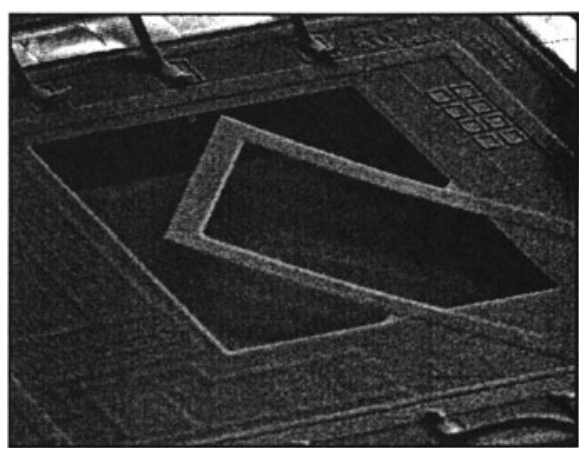

Figure 11: The sensing system after FSBM post-process.

\section{CONCLUSION}

An advantage of monolithic integration is the possibility to add electronic functions without significant increase of the total system cost. The U-Shape cantilever performance in the magnetometer application does not reach the performance level of sensors built on dedicated technologies in term of sensitivity. Although, the use of polysilicon resistances gauges in a Wheatstone bridge structure offers many advantages such:

- Effect of temperature on the gauge is cancelled,

- Noise power spectral density in resistances is low and good resolutions are achievable with appropriate signal processing,

- It is easy to interface with electronics.

After amplification and noise filtering, the sensor resolution has been taken to $2 \mu \mathrm{T}$, giving the system the ability to measure earth magnetic field.

Further improvements of the signal processing circuit will implement oversampling techniques in order to reduce noise level. 


\section{REFERENCES}

[1] G.Rieger, K. Ludwig, J. Hauch, W. Clemens, "GMR sensors for contactless position detection", Sensors and Actuators A 91 (2001) 7-11.

[2a] L. Latorre, Y. Bertrand, P. Nouet, "On the use of Test Structures for the Electromechanical characterization of a CMOS compatible MEMS technology", Proc. IEEE 1998 Int. Conf. On Microelectronic Test Structures, March, 1998.

[2b] L. Latorre, P. Nouet, Y. Bertrand, P. Hazard and F.Pressecq, "Characterization and modeling of a CMOS-compatible MEMS technology", Sensors and Actuators, Vol. 74, 1999, pp. 143-147.

[2c] L.Latorre, V. Beroulle, Y. Bertrand, I. Salesse, P. Nouet, « Electro-Mechanical Magnetic Field sensors in a CMOS Technology », Proc. of DCIS 2000, pp694-699, 15 ${ }^{\text {th }}$ Design of Circuits and Integrated Systems Conference, Montpellier, Le Corum, France, November 21-24, 2000.

[2d] V. Beroulle, Y. Bertrand, L. Latorre, P. Nouet, « Micromachined CMOS Magnetic Field Sensors with Low-Noise Signal Conditioning », $15^{\text {th }}$ IEEE INTERNATIONAL MICRO ELECTRO-MECHANICAL SYSTEMS CONFERENCE, January 20-24, 2002, Las Vegas, Nevada, USA.

[3] Circuits Multi-projets (CMP) is a broker in Ics, MCMs and MEMS. See http://cmp.imag.fr/

[4] L. Latorre and al., "Characterization and modeling of a CMOS-compatible MEMS technology", Sensors and Actuators, Vol. 74, 1999, pp. 143-147.

[5] Jonah A. Harley, "Advances in piezoresistive probes for atomic force microscopy", Dissertation of Ph'D degree, march 2000.

[6] R. S. Popovic, « Hall effect Devices, Magnetic Sensors and Characterization of semiconductors », The Adam Hilger Series On Sensors, Adam Hilger, ISBN 0-75030096-5, 1991

[7] See http://www.ams.co.at 